\title{
Location-based admission control for differentiated services in $3 G$ cellular networks
}

\author{
Rudesindo Núñez-Queija ${ }^{*}$ \\ Department of Mathematics and Computer \\ Science, Eindhoven University of Technology \\ 5600 MB Eindhoven \\ The Netherlands \\ sindo@cwi.nl
}

\author{
Hwee-Pink $\operatorname{Tan}^{\dagger}$ \\ Center for Telecommunications Value-Chain \\ Research \\ Trinity College, University of Dublin \\ Dublin 2, Ireland \\ tanhp@tcd.ie
}

\begin{abstract}
Third generation wireless systems can simultaneously accommodate flow transmissions of users with widely heterogeneous applications. As resources are limited (particularly in the air interface), admission control is necessary to ensure that all active users are accommodated with sufficient capacity to meet their specific Quality of Service requirements. Our admission control rule protects users with stringent capacity requirements ("streaming traffic") while offering sufficient capacity over longer time intervals to delay-tolerant users ("elastic traffic"). Performance evaluation of wireline differentiated-services platforms is already difficult due to the inherently large dimensionality of models to capture the diversity of user applications. In wireless systems, this is further exemplified as the location of users adds to the dimensionality problem. Using time-scale decomposition, we develop approximations to evaluate the performance of a differentiated admission control strategy to support integrated services with capacity requirements in a realistic downlink transmission scenario for a single radio cell.
\end{abstract}

Categories and Subject Descriptors: I.6.4 Simulation and Modeling: Model Validation and Analysis.

General Terms: Performance, Design.

Keywords: Admission control, differentiated services, 3G cellular networks.

\section{INTRODUCTION}

Third Generation (3G) cellular networks such as UMTS and CDMA2000 are expected to support a large variety of applications, where the traffic they carry is commonly grouped into two broad categories: Elastic traffic corre-

\footnotetext{
${ }^{*}$ Currently affiliated with CWI and TNO Information and Communication Technology (The Netherlands).

${ }^{\dagger}$ At the time of this research, this author was affiliated with EURANDOM (The Netherlands).
}

Permission to make digital or hard copies of all or part of this work for personal or classroom use is granted without fee provided that copies are not made or distributed for profit or commercial advantage and that copies bear this notice and the full citation on the first page. To copy otherwise, to republish, to post on servers or to redistribute to lists, requires prior specific permission and/or a fee.

MSWiM'06, October 2-6, 2006, Torremolinos, Malaga, Spain.

Copyright 2006 ACM 1-59593-477-4/06/0010 ...\$5.00. sponds to the transfer of digital documents (e.g., Web pages, emails, stored audio / videos) characterized by their size, i.e., the volume to be transferred. Applications carrying elastic traffic are flexible, or "elastic", towards capacity fluctuations, the total transfer time being a typical performance measure. Streaming traffic corresponds to the real-time transfer of various signals (e.g., voice, streaming audio / video) characterized by their duration as well as their transmission rate. Stringent capacity guarantees are necessary to ensure real-time communication to support applications carrying streaming traffic.

Various papers have been published recently that study wired links carrying integrated (elastic and streaming) traffic. In terms of resource sharing, the classical approach is to give head-of-line priority to packets of streaming traffic in order to offer packet delay and loss guarantees [1, 9, 14]; alternatively, adaptive streaming traffic (that are TCP-friendly and mimic elastic traffic) is considered in $[4,12,11]$. Markovian models have been developed for the exact analysis of these systems [14, 13]. However, they can be numerically cumbersome due to the inherently large dimensionality required to capture the diversity of user applications. Hence, various approximations have been proposed [1, 11], where the obtained closed-form limit results can serve as performance bounds, and hence yield useful insight.

In this study, we consider downlink transmissions of integrated traffic in a single $3 \mathrm{G}$ radio cell and propose an admission control strategy that allocates priority to streaming traffic through resource reservation and guarantees the capacity requirements of all users while maximizing the data rate of each elastic user. The location-dependence of the wireless link capacity adds to the dimensionality problem already inherent in the performance analysis of corresponding wireline integrated services platforms. In our previous work [7], we disregard the location of the users in the admission control model by assuming that all users are located at the cell border, consuming more resource than they actually do. As a result, fewer users can be admitted, giving rise to a conservative admission control model. Here, we generalize the model by taking into account the location of each user to achieve a more realistic representation of the actual scenario. We describe the model in Section 2 and develop an approximation based on time-scale decomposition in Section 3. Numerical results are presented in Section 4. Some concluding remarks are outlined in Section 5 .

\section{MODEL}


We consider a $3 \mathrm{G}$ radio cell (e.g., UMTS/W-CDMA) with a single downlink channel whose transmission power at the base station (resource) is shared amongst users carrying streaming and elastic traffic. We assume that the base station transmits at full power denoted by $P$ (see Footnote 1 ), where a part of it, $P_{s} \leq P$, is statically reserved for streaming traffic, and unclaimed power is equally shared amongst all elastic users. Note that although the resource that can be maximally guaranteed for on-going elastic traffic is $P_{e}$ $=P-P_{s}$, they are permitted to use more than $P_{e}$. However, the surplus is immediately allocated to streaming traffic when a new streaming user arrives.

With W-CDMA technology, the base station can transmit to multiple users simultaneously using orthogonal code sequences. Let $P_{u} \leq P$ be the power transmitted to user $u$ located at distance $\delta_{u}$ from its serving base station. The power received by user $u$ is $P_{u}^{r}=P_{u} \Gamma_{u}$, where $\Gamma_{u}$ denotes the attenuation due to path-loss. For typical radio propagation models, $\Gamma_{u}$ is proportional to $\left(\delta_{u}\right)^{-\gamma}$, where $\gamma$ is a positive path-loss exponent.

The quality of the received signal at user $u$ is specified in terms of the energy-per-bit to noise-density ratio, $\left(\frac{E_{b}}{N_{0}}\right)_{u}$ :

$$
\left(\frac{E_{b}}{N_{0}}\right)_{u}=\frac{W}{R_{u}} \frac{P_{u}^{r}}{\eta+I_{u}^{a}+I_{u}^{r}}
$$

where $W$ is the CDMA chip rate, $R_{u}$ is the instantaneous data rate of user $u, \eta$ is the background noise (assumed to be constant throughout the cell) and $\left(I_{u}^{a}, I_{u}^{r}\right)$ is the intr $a /$ inter-cell interference at user $u$ respectively. As the name suggests, intra (inter)-cell interference is caused by simultaneous interfering transmissions received at user $u$ from the base station in the serving cell (neighboring cells). Intracell interference at user $u, I_{u}^{a}$, arises due to simultaneous transmissions from user $u$ 's serving base station using nonorthogonal codes (with total power $P_{u}^{a}$ ) to other users in the same cell received at user $u$. Quantitatively, $I_{u}^{a}=\alpha P_{u}^{a} \Gamma_{u}$, where $\alpha$ is the code non-orthogonality factor.

To achieve a target error probability corresponding to a given Quality of Service (QoS), it is necessary that $\left(\frac{E_{b}}{N_{0}}\right)_{u} \geq$ $\epsilon_{u}$, for some threshold $\epsilon_{u}$. Equivalently, the data rate $R_{u}$ of each admitted user $u$ is upper-bounded as follows:

$$
R_{u} \leq \frac{W P_{u} \Gamma_{u}}{\epsilon_{u}\left(\eta+\alpha P_{u}^{a} \Gamma_{u}+I_{u}^{r}\right)} .
$$

\subsection{Resource Sharing}

The total interference power, $P_{u}^{a}$, with respect to user $\mathrm{u}$ depends on how the base station power, $P$, is shared amongst all users (i.e., the resource sharing mode):

$$
P_{u}^{a} \begin{cases}=P-P_{u}, & \text { simultaneous transmission to } \\
<P-P_{u}, & \begin{array}{l}
\text { all users in the cell; } \\
\text { simultaneous transmission to } \\
\text { some users in the cell; }
\end{array} \\
=0, & \begin{array}{l}
\text { no simultaneous transmission } \\
\text { (time-sharing }) .
\end{array}\end{cases}
$$

Based on our definition in Section 1, each streaming (elastic) user $u$ has a fixed (minimum) capacity requirement, denoted by $r_{u}$. According to our resource reservation policy, while each streaming user transmits at fixed rate $r_{u}$, the transmission rate of an elastic user $u, R_{u}\left(\geq r_{u}\right)$, depends on the resource unclaimed by streaming traffic. From Eq.
(1), $R_{u}$ can be maximized by minimizing $P_{u}^{a}$, i.e., by applying time-sharing amongst elastic users.

If we aggregate all elastic users into one fictitious user, the resource sharing mechanism is such that the base station transmits using (almost-) orthogonal codes to all users. Within the aggregate user, elastic users sharing the same code are served in a time-slotted fashion so that they do not interfere with one another, but only with elastic users using different codes and streaming traffic. This resource sharing mode is similar to UMTS / HSDPA, where up to $N_{c}=4$ codes can be shared amongst data/elastic users. We assume that $N_{c}=1$ in our study, i.e., while the received signal for a streaming user is interfered by simultaneous transmissions to all other users, that for an elastic user is interfered by simultaneous transmissions to streaming users only.

\subsection{Cell Partitioning}

From Eq. (1), the transmission power required to support the capacity requirement, $r_{u}$, of user $u$ is given by:

$$
P_{u} \geq \frac{r_{u} \epsilon_{u}\left[\alpha P_{u}^{a} \Gamma_{u}+\eta+I_{u}^{r}\right]}{W \Gamma_{u}} \equiv \tilde{P}_{u} .
$$

Ideally, given the exact location of each user $u$, a maximum number of users can be admitted if the base station allocates exactly $\tilde{P}_{u}$ to each user $u$. However, for our analysis to be tractable, it is necessary to quantize the location of each user in the cell. We do so by dividing the cell into $J$ disjoint segments, where we assume that the path-loss, intracell and inter-cell interference are the same for any user in segment $j=1, \ldots, J$, denoted by $\left(\Gamma_{j}, I_{j}^{a}, I_{j}^{r}\right)$, respectively. As $J$ increases, the location quantization becomes finer and approaches the ideal case $(J=\infty)$.

Accordingly, we assume that type- $x$ users arrive at segment $j$ as independent Poisson processes at rate $\lambda_{j, x}$ with capacity requirement of $r_{j, x}>0$. Elastic users in segment $j$ have a general file size (or service requirement) distribution with mean $f_{j, e}$ (bits) and, similarly, the holding times of streaming users may be taken to have mean $1 / \mu_{j, s}$ (secs). The total arrival rate of type- $x$ users to the cell is denoted by $\lambda_{x}=\sum_{j=1}^{J} \lambda_{j, x}$. The minimum energy-to-noise ratio, $\epsilon_{u}$, may depend on the user type and location [10], and will be denoted by $\epsilon_{j, x}$ for type- $x$ users in segment $j$.

\subsection{Admission Control}

We propose an admission control strategy that ensures the required capacity $r_{u}$ of each admitted user $u$ is satisfied. Let $N_{j, x}$ denote the number of type- $x$ users in segment $j$, and define $N_{j}=N_{j, e}+N_{j, s}$. We further define the vector $\mathbf{N}_{x}=$ $\left(N_{1, x}, \ldots, N_{J, x}\right)$ and let $N_{x}$ be the total number of type- $x$ users in the cell. Let $\left(\beta_{j}, \gamma_{j}\right)$ be the minimum transmission power required by an (elastic, streaming) user in segment $j$ to sustain a capacity requirement of $\left(r_{j, e}, r_{j, s}\right)$, respectively.

According to our resource sharing policy, the received signal at each streaming user $u$ in segment $j$ is interfered by simultaneous transmissions to all other users, i.e., $P_{u}^{a}=P$ $P_{u}$ and from (2) we obtain

$$
r_{j, s} \epsilon_{j, s}\left[\alpha\left(P-P_{j, s}\right) \Gamma_{j}+\eta+I_{j}^{r}\right] \leq W P_{j, s} \Gamma_{j},
$$

so that $\gamma_{j}=\frac{r_{j, s} \epsilon_{j, s}\left[\alpha P \Gamma_{j}+\eta+I_{j}^{r}\right]}{\left(W+\alpha r_{j, s} \epsilon_{j, s}\right) \Gamma_{j}}$. Streaming users are always accommodated with exactly their required capacity, consuming a total power of $P_{s}\left(\mathbf{N}_{s}\right)=\sum_{j=1}^{J} N_{j, s} \gamma_{j}{ }^{1}$.

\footnotetext{
${ }^{1}$ When only streaming users are active, there exists a power
} 
For an elastic user $u$ in segment $j$, we have $P_{u}^{a}=P_{s}\left(\mathbf{N}_{s}\right)$ since its received signal is only interfered by streaming users. Hence, the power required by an elastic user in segment $j$ to sustain its capacity requirement, $r_{j, e}$, depends on the number and location of streaming users as follows:

$$
\beta_{j}\left(\mathbf{N}_{s}\right)=\frac{r_{j, e} \epsilon_{j, e}\left[\alpha P_{s}\left(\mathbf{N}_{s}\right) \Gamma_{j}+\eta+I_{j}^{r}\right]}{W \Gamma_{j}} .
$$

The admission control scheme is such that a newly-arrived user is blocked only if accepting it would violate either the static reservation policy or the minimum power requirement of any user. At any time, streaming traffic can claim a portion $P_{s}$ of the total power $P$. Therefore, the power required by an elastic user in segment $j$ is at least

$$
\beta_{j}=\frac{r_{j, e} \epsilon_{j, e}\left[\alpha P_{s} \Gamma_{j}+\eta+I_{j}^{r}\right]}{W \Gamma_{j}} .
$$

Note that $\beta_{j}$ is insufficient to guarantee capacity $r_{j, e}$ if streaming traffic consumes more than $P_{s}$. In contrast, $\gamma_{j}$ is always sufficient to achieve rate $r_{j, s}$.

The capacity of elastic users must be achievable with power $P_{e}=P-P_{s}$. Since all elastic users receive an equal portion of the available power, we conclude that $N_{e} \beta_{j} \leq P_{e}$ must hold for all $j$ with $N_{j, e}>0$, or equivalently,

$$
N_{e} \beta_{j} \mathbf{1}_{\left(N_{j, e}>0\right)} \leq P_{e}, \quad \forall j .
$$

While we assume here a simple round-robin scheduler for the elastic users' transmissions for modeling simplicity, higher data rates can be achieved for these users with an opportunistic scheduler (e.g., proportionally-fair). This can be captured by dividing the factor $N_{e}$ by an increasing gain function, $\mathrm{G}\left(N_{e}\right)$ [5] without introducing additional modeling complexity (See [6] for details).

The indicator function $\mathbf{1}_{E}$ equals 1 if expression $E$ holds and is 0 otherwise. Note that the $J$ conditions in (4) only limit the total number of elastic users $N_{e}$, but that the maximum number of users does depend on the entire vector $\mathbf{N}_{e}$. Similarly, the fact that elastic users share power equally, together with the minimum power restrictions of both elastic and streaming users, imply that

$$
N_{e} \beta_{j}\left(\mathbf{N}_{s}\right) \mathbf{1}_{\left(N_{j, e}>0\right)}+P_{s}\left(\mathbf{N}_{s}\right) \leq P, \quad \forall j .
$$

It is worth noting that the functions $\beta_{j}\left(\mathbf{N}_{s}\right)$ and $P_{s}\left(\mathbf{N}_{s}\right)$ depend only on $\mathbf{N}_{s}$ through the weighted sum $\sum_{j=0}^{J} N_{j, s} \gamma_{j}$.

Conditions (4) and (5) completely determine the admission policy: a newly-arrived user will be accepted only if the resulting system state, $\left(\mathbf{N}_{e}, \mathbf{N}_{s}\right)$, satisfies all $2 J$ conditions. Alternatively, these conditions may be formulated in terms of the required power for each user type. Similar to $P_{s}\left(\mathbf{N}_{s}\right)$, we determine the transmission power required by elastic requests, noting its dependence on the system state:

$$
P_{e}\left(\mathbf{N}_{e}, \mathbf{N}_{s}\right) \equiv N_{e} \times \max _{j: N_{j, e}>0}\left\{\beta_{j}\left(\mathbf{N}_{s}\right)\right\}
$$

Our admission control policy for streaming users can now be formulated as follows: a newly-arrived streaming user in

level $P^{\prime}<P$ for which Eq. (3) holds, thus achieving power savings. Conversely, if $P$ ' exists such that Eq. (3) holds, then Eq. (3) necessarily holds when transmitting at full power (increase all transmit powers proportionally until full power is used). From a modeling perspective, we can therefore assume that the base station transmits at full power. segment $i$ will be admitted if

$$
P_{e}\left(\mathbf{N}_{e}, \mathbf{N}_{s}+\mathbf{e}_{i}\right)+P_{s}\left(\mathbf{N}_{s}+\mathbf{e}_{i}\right) \leq P,
$$

where the vector $\mathbf{e}_{i}$ has its $i^{\text {th }}$ component equal to 1 and all other components are 0 .

For elastic users, we must incorporate the power reservation restrictions as well. We define

$$
\bar{P}_{s}\left(\mathbf{N}_{s}\right) \equiv \max \left\{P_{s}, P_{s}\left(\mathbf{N}_{s}\right)\right\}
$$

and

$$
\bar{P}_{e}\left(\mathbf{N}_{e}, \mathbf{N}_{s}\right) \equiv N_{e} \times \max _{j: N_{j, e}>0}\left\{\max \left\{\beta_{j}, \beta_{j}\left(\mathbf{N}_{s}\right)\right\}\right\} .
$$

Taking the maximum of $\beta_{j}$ and $\beta_{j}\left(\mathbf{N}_{s}\right)$ ensures that if streaming traffic uses less than the reserved capacity, i.e., $P_{s}\left(\mathbf{N}_{s}\right)<$ $P_{s}$, the minimum capacity requirement for elastic users in segment $j$ can be guaranteed, even if streaming traffic claims the full reserved power at a later stage. Hence, a newlyarrived elastic user in segment $i$ will be admitted if

$$
\bar{P}_{e}\left(\mathbf{N}_{e}+\mathbf{e}_{i}, \mathbf{N}_{s}\right)+\bar{P}_{s}\left(\mathbf{N}_{s}\right) \leq P .
$$

While the admission control proposed in [1] is similar, it results in equal blocking probabilities for both types of traffic. Due to resource reservation in our case, the blocking probabilities will depend on both the user type and location. In addition, our model simplifies to the conservative model defined in [7] for $J=1$ if time-sharing is disabled.

\subsection{Rate allocation}

As mentioned above, streaming users are accommodated with exactly their required capacities, i.e., $r_{j, s}$ in segment $j$. For elastic users, the rates depend on the number, type and location of other users. The available transmission power for elastic flows is $P-P_{s}\left(\mathbf{N}_{s}\right)$, of which all active elastic users receive an equal portion. Using (2), an elastic user in segment $j$ attains a data rate

$$
r_{j, e}\left(N_{e}, \mathbf{N}_{s}\right)=\frac{1}{N_{e}} f_{j, e} \mu_{j, e}\left(\mathbf{N}_{s}\right),
$$

where

$$
\mu_{j, e}\left(\mathbf{N}_{s}\right)=\frac{1}{f_{j, e}} \times \frac{W\left(P-P_{s}\left(\mathbf{N}_{s}\right)\right) \Gamma_{j}}{\epsilon_{j, e}\left[\alpha P_{s}\left(\mathbf{N}_{s}\right) \Gamma_{j}+\eta+I_{j}^{r}\right]}
$$

can be interpreted as the total departure rate of elastic users if all elastic users are in segment $j$.

\section{ANALYSIS}

Since exact analysis of our model is non-tractable in general, we develop an approximation based on time-scale decomposition to evaluate the cell performance and assess the accuracy through comparison with simulation.

\subsection{Time-scale Decomposition}

Time-scale decomposition is a technique that exploits the difference in time-scale of the relative dynamics of each traffic type to isolate them so that analysis becomes tractable. For example, if the dynamics of streaming (elastic) flows take place on a much slower time scale than those of elastic flows, then, elastic (streaming) traffic practically reaches statistical equilibrium while the number of active streaming (elastic) users remain unchanged. Under this assumption, the dynamics of elastic (streaming) flows can be modeled by an egalitarian processor-sharing queue (Erlang-loss 
queue). Since closed-form expressions exist for the solution of these well-known queueing models, performance metrics to approximate the cell performance can be derived.

Accordingly, we define two approximations (termed quasistationary and fluid respectively) corresponding to each traffic regime presented in the preceding example. We describe the development of the quasi-stationary approximation, $\mathbf{A}(\mathbf{Q})$ in detail here; a detailed description of the complementary fluid approximation, $\mathbf{A}(\mathbf{F})$ can be found in [6].

\subsection{Quasi-stationary Approximation}

To apply the quasi-stationary approximation, we consider the combination of voice calls (streaming) and web-browsing or email (elastic) applications, where the dynamics of streaming flows take place on a much slower time scale than those of elastic flows, i.e., all $\mu_{j, s}$ and $\lambda_{j, s}$ are much smaller than any of the quantities $1 / f_{j, e}$ and $\lambda_{j, e}$.

\subsubsection{Conditional distribution for elastic traffic}

With the above assumption, the number of active elastic flows instantaneously reaches a new statistical equilibrium whenever $\mathbf{N}_{s}$ changes. Hence, for fixed $\mathbf{N}_{s} \equiv \mathbf{n}_{s}$, the elastic traffic behaves like a $J$-class $M / G / 1$ processor-sharing (PS) queue with admission control dictated by both (4) and (5).

For general service requirement distributions of elastic users and an admission region of the type $\sum_{j} N_{j, e} \leq M$, the steady state distribution of the numbers of users in each segment was shown to be a multivariate geometric distribution [8]. This can be shown to imply the same stationary distribution (up to a multiplicative constant) for the elastic users under the quasi-stationary assumption. For phasetype distributions, this can be proved formally by taking $M$ large enough so that the set of allowable states (4) and (5) can be included. The joint process of queue lengths and service phases is reversible, so that state-space truncation does not destroy detailed balance and one can obtain the stationary distribution of the restricted process by re-normalization of the steady-state measure:

$$
\begin{aligned}
\mathbb{P}\left(\mathbf{n}_{e} \mid \mathbf{n}_{s}\right) & \equiv \mathbb{P}\left(\mathbf{N}_{e}=\mathbf{n}_{e} \mid \mathbf{N}_{s}=\mathbf{n}_{s}\right) \\
& =c_{e}\left(\mathbf{n}_{s}\right) n_{e} ! \prod_{j=1}^{J} \frac{\rho_{j, e}\left(\mathbf{n}_{s}\right)^{n_{j, e}}}{n_{j, e} !},
\end{aligned}
$$

where $\rho_{j, e}\left(\mathbf{n}_{s}\right)=\frac{\lambda_{j, e}}{\mu_{j, e}\left(\mathbf{n}_{s}\right)}$ and the normalization constant $c_{e}\left(\mathbf{n}_{s}\right)$ is such that adding (8) over all $\mathbf{n}_{e}$ that satisfy (4) and (5) gives a total of 1 , for each fixed $\mathbf{n}_{s}$. We finally recall that $n_{e}=\sum_{j=1}^{J} n_{j, e}$.

The conditional acceptance probability of newly-arrived elastic flows in segment $i$, equals

$$
A_{i, e}\left(\mathbf{n}_{s}\right) \equiv \mathbb{P}\left(\bar{P}_{e}\left(\mathbf{N}_{e}+\mathbf{e}_{i}, \mathbf{n}_{s}\right) \leq P-\bar{P}_{s}\left(\mathbf{n}_{s}\right) \mid \mathbf{N}_{s}=\mathbf{n}_{s}\right) .
$$

From (8) we can also obtain the distribution of the total number of active elastic users by summing over all admitted combinations of $n_{j, e}$ with $\sum_{j} n_{j, e}=n_{e}$. For the special case where $\beta_{i} \equiv \beta$ for all $i-$ we call this uniform admission control $^{2}-$, the distribution for the total number of elastic

${ }^{2}$ With uniform admission control, the minimum required power is the same for all users, irrespective of their locations. Consequently, users further from the base station (with larger inter-cell interference) must compromise for a users reduces to a simple truncated geometric distribution:

$$
\mathbb{P}\left(N_{e}=n_{e} \mid \mathbf{N}_{s}=\mathbf{n}_{s}\right)=\frac{\left(1-\rho_{e}\left(\mathbf{n}_{s}\right)\right) \rho_{e}\left(\mathbf{n}_{s}\right)^{n_{e}}}{1-\rho_{e}\left(\mathbf{n}_{s}\right)^{n_{e}^{\max }\left(\mathbf{n}_{s}\right)+1}},
$$

where $n_{e}^{\max }\left(\mathbf{n}_{s}\right)=\left\lfloor\left(P-\bar{P}_{s}\left(\mathbf{n}_{s}\right) / \beta\right)\right\rfloor$.

We emphasize that Eq. (8) and (9) are valid for general file size distributions of elastic requests [8], depending on this parameter only through its mean. As a further remark, often, when applying a time-scale decomposition, the issue of stability is of considerable importance, giving rise to an additional assumption commonly referred to as uniform stability [9]. However, this is of no concern in our model, since $\mathbf{N}_{e}$ is bounded due to the assumption that $r_{j, e}>0$.

\subsubsection{Unconditional marginal distributions}

Next, we consider the dynamics of streaming flows. When $\mathbf{N}_{s}=\mathbf{n}_{s}$, streaming flows depart at a rate $\sum_{j} n_{j, s} \mu_{j, s}$. When a new streaming flow arrives in segment $i$, the probability of acceptance, $A_{i, s}\left(\mathbf{n}_{s}\right)$, is given by:

$$
\mathbb{P}\left(P_{e}\left(\mathbf{N}_{e}, \mathbf{n}_{s}+\mathbf{e}_{i}\right) \leq P-P_{s}\left(\mathbf{n}_{s}+\mathbf{e}_{i}\right) \mid \mathbf{N}_{s}=\mathbf{n}_{s}\right) .
$$

Hence, the effective arrival rate of streaming flows in segment $i, \Lambda_{i, s}\left(\mathbf{n}_{s}\right)$, is given as follows:

$$
\Lambda_{i, s}\left(\mathbf{n}_{s}\right)=\lambda_{i, s} A_{i, s}\left(\mathbf{n}_{s}\right) .
$$

In general, there is no closed-form expression for the equilibrium distribution of $\mathbf{N}_{s}$ and we must assume exponential or phase-type holding time distributions and resort to standard methods to (numerically) solve the equilibrium distribution of a finite-state Markov process. Note that the dimension of this process $\mathbf{N}_{s}$ is much smaller than the original process $\left(\mathbf{N}_{e}, \mathbf{N}_{s}\right)$ : the component $\mathbf{N}_{e}$ is "eliminated" in the approximation. However, if we apply uniform admission control for streaming traffic by taking $\gamma_{j} \equiv \gamma$ independent of $j$ [see the earlier Footnote 2], then $A_{i, s}\left(\mathbf{n}_{s}\right) \equiv A_{s}\left(n_{s}\right)$ is independent of $i$ and depends on $\mathbf{n}_{s}$ only through the total number of streaming flows. $\mathbf{N}_{s}$ can then be shown to be balanced [2] and can be reduced to the framework of [8]. It follows that, for arbitrary holding time distributions of streaming flows, and $0 \leq n_{s} \leq n_{s}^{\max }=\left\lfloor\frac{P}{\gamma}\right\rfloor$ :

$$
\mathbb{P}\left(\mathbf{N}_{s}=\mathbf{n}_{s}\right)=c_{s} \prod_{k=0}^{n_{s}-1} A_{s}(k) \prod_{j=1}^{J} \frac{\left(\rho_{j, s}\right)^{n_{j, s}}}{n_{j, s} !}
$$

with $\rho_{j, s}=\lambda_{j, s} / \mu_{j, s}$ and $c_{s}=P\left(N_{s}=0\right)$ can be determined by normalizing (10) to a probability distribution. Letting $\rho_{s}=\sum_{j} \rho_{j, s}$, we obtain the distribution of $N_{s}$ (still for uniform admission control):

$$
\mathbb{P}\left(N_{s}=n_{s}\right)=c_{s} \frac{\left(\rho_{s}\right)^{n_{s}}}{n_{s} !} \prod_{k=0}^{n_{s}-1} A_{s}(k),
$$

which in this case results again in a simple expression for the normalizing constant:

$$
c_{s}=\left(\sum_{n_{s}=0}^{n_{s}^{\max }} \frac{\left(\rho_{s}\right)^{n_{s}}}{n_{s} !} \prod_{k=0}^{n_{s}-1} A_{s}(k)\right)^{-1} .
$$

lower rate. Thus, although the admission policy is the same, users in different segments are distinguished by the achievable rates (as well as their own traffic distributions). 


\begin{tabular}{|c|c|}
\hline \multicolumn{2}{|c|}{ UMTS and traffic parameters } \\
\hline$P(\mathrm{~W})$ & $(20,0.2)$ \\
\hline $\mathrm{P}_{\mathrm{s}}(\mathrm{W})$ & 10 \\
\hline$\eta(\mathrm{W})$ & $6.09 \times 10^{-14}$ \\
\hline$W(\mathrm{chips} / \mathrm{s})$ & $3.84 \times 10^{6}$ \\
\hline$\varepsilon(\mathrm{dB})$ & 2 \\
\hline$\alpha$ & 0.5 \\
\hline Propagation Model & Okumura-Hata Model [16] \\
\hline Inter-cell Interference & $\begin{array}{c}\text { Hexagonal network with } \\
\text { maximum tx. power [3] }\end{array}$ \\
\hline Model & Table 8.3 [10] \\
\hline Link budget & 128 \\
\hline$r_{e}(\mathrm{kbps})$ & 128 \\
\hline$r_{s}(\mathrm{kbps})$ & \\
\hline
\end{tabular}

Table 1: UMTS cell and traffic parameters for performance evaluation.

To conclude this section, we now calculate several relevant performance measures (not restricting anymore to uniform admission control) by un-conditioning on $\mathbf{N}_{s}$. The unconditional distribution for the number of elastic users is

$$
\mathbb{P}\left(\mathbf{N}_{e}=\mathbf{n}_{e}\right)=\sum_{\mathbf{n}_{s}} \mathbb{P}\left(\mathbf{n}_{e} \mid \mathbf{n}_{s}\right) \mathbb{P}\left(\mathbf{N}_{s}=\mathbf{n}_{s}\right),
$$

and a type- $x$ user in segment $i$ is blocked with probability

$$
p_{i, x}=\sum_{\mathbf{n}_{s}}\left(1-A_{i, x}\left(\mathbf{n}_{s}\right)\right) \mathbb{P}\left(\mathbf{N}_{s}=\mathbf{n}_{s}\right) .
$$

\section{PERFORMANCE EVALUATION}

We consider a single UMTS cell whose radius, $\delta_{J}$, is computed using the reference link budget given in Table 8.3 [10] and the Okumura-Hata propagation model [16] for an urban macro cell. The inter-cell interference at each location within the cell is computed based on the conservative approximation for a hexagonal network [3].

Each elastic (streaming) user arrives at the cell with file size, $s_{e}$ (holding time, $d_{s}$ ) with mean $f_{e}\left(\frac{1}{\mu_{s}}\right)$. The base station performs admission control according to the type and location of each user, assumed to be uniformly distributed over the cell. Unless otherwise stated, we assume that $\left(s_{e}\right.$, $d_{s}$ ) follow an exponential distribution (Case $\mathbf{I}$ ).

In addition to the mean number of users, $\left(\mathrm{E}\left[N_{e}\right], \mathrm{E}\left[N_{s}\right]\right)$, and blocking probabilities, $\left(p_{e}, p_{s}\right)$, for each class of traffic, we define the stretch, $S_{e}$, for each admitted elastic user as the normalized expected residence time, $E\left[R_{e}\right]$, i.e., $S_{e}=$ $\frac{E\left[R_{e}\right]}{f_{e}}=\frac{E\left[N_{e}\right]}{f_{e} \lambda_{e}\left(1-p_{e}\right)}$ (cf. Little's Theorem). A summary of the cell and traffic parameters is given in Table 1.

\subsection{Simulation Procedure}

We develop a simulation program for our model by considering arrival / departure events of traffic requests (elastic or streaming). Each simulation scenario is defined according to the following procedure:

1. Fix the level of location quantization, $J$ :

$J=1$ : no location information;

$J>1$ : location information available.

2. Fix the total offered traffic load by choosing $l>0$, where $u_{e}+u_{s}=l c, u_{e}=\lambda_{e} f_{e}$ and $u_{s}=\frac{\lambda_{s} r_{s}}{\mu_{s}} ;$ 3. Fix the traffic mix, $\frac{u_{e}}{l c}$, by choosing $u_{e}, 0 \leq u_{e} \leq l \stackrel{\mu_{s}}{\text {; }}$ 4. Select $\left(\lambda_{e}, \lambda_{s}\right)$ to fit a selected traffic regime.

\subsection{Performance Insensitivity with Traffic Pa- rameter Distribution}

We begin by investigating the sensitivity of the cell performance towards traffic parameter distribution for $J=1$. Hence, in addition to Case $\mathbf{I}$, we define the following additional cases: II with exponentially distributed $d_{s}$ and hyperexponentially distributed $s_{e}$ with parameter $a_{e}$ (cf.[15], p. 359), where

$$
P\left(s_{e}>s\right)=\frac{a_{e} e^{\frac{-a_{e} s}{f_{e}}}+e^{\frac{-s}{a_{e} f_{e}}}}{a_{e}+1}, \forall s
$$

and

$$
\operatorname{Var}\left[s_{e}\right]=\left(a_{e}+\frac{1}{a_{e}}-1\right) f_{e}^{2} .
$$

and III with exponentially distributed $s_{e}$ and Erlang- $k$ distributed $d_{s}$, where $\forall d \geq 0$ and $k>0$,

$$
f_{s}(d)=\frac{k \mu_{s}\left(k \mu_{s} d\right)^{k-1}}{(k-1) !} e^{-k \mu_{s} d}
$$

and $\operatorname{Var}\left[d_{s}\right]=\frac{1}{k \mu_{s}^{2}}$.

We generate 5 sets of simulation results for each scenario, and compute the sample mean for $\left(p_{e}, p_{s}, S_{e}\right)$. The results are tabulated in Table 2 for $a_{e}=100$ (II) and $k=2$ (III) for a fully-loaded cell (i.e., $l=1$ ). We observe that the performance measures obtained for Cases II and III are within $10 \%$ of those obtained for Case I. Hence, the performance is almost insensitive to traffic parameter distributions, justifying the proposed insensitive approximations.

\subsection{Accuracy of Quasi-stationary Approxima- tion}

To apply the quasi-stationary approximation to estimate the cell performance analytically, we define each segment $j$ as the annulus between concentric rings of radius $\delta_{j-1}$ and $\delta_{j}$ such that $\delta_{j}=\frac{j}{J} \delta_{J}, 1 \leq j \leq J$, where the arrival rate of users in each ring $j$ is $\lambda_{j}=\frac{\delta_{j}^{2}-\delta_{j-1}^{2}}{\delta_{J}^{2}} \lambda$, where $\delta_{0}=0$, due to the assumption of uniformly distributed arrivals. To apply Eq. (10) and Eq. (11) for general holding time distributions, we assume uniform admission control for streaming traffic by taking $\gamma_{j}=\gamma_{J}$ [see Footnote 2].

We investigate the accuracy of the approximation for various values of $J$ (denoted by $\mathbf{A}(\mathbf{Q}, \mathbf{J})$ ) by bench-marking against simulation results (Case $\mathbf{I}$ ) obtained in Section 4.2 . We plot $\left(p_{e}, p_{s}\right)$ as a function of the traffic mix, $\frac{u_{e}}{c}, 0 \leq u_{e} \leq$ $c$, for $\mathbf{A}(\mathbf{Q}, \mathbf{J})$ in Fig. 1. We observe that, for $P=20 \mathrm{~W}$, the cell performance obtained with simulation is well approximated by $\mathbf{A}(\mathbf{Q}, \mathbf{J}=\mathbf{1})$, and that $\mathbf{A}(\mathbf{Q}, \mathbf{J})$ is almost invariant with the value of $J$. Although cell partitioning (with increasing $J$ ) was intended to improve the accuracy of the approximations by reducing the quantization error of estimating each user's location, for the given base station transmission power, the cell performance can be well approximated using the conservative admission control in [7], which does not exploit user location. However, with $P=20 \mathrm{~W}$, this could be expected since the signal-to-interference noise ratio remains relatively constant before falling steeply beyond the cell edge. 


\begin{tabular}{|c|c|c|c|c|c|c|c|c|c|c|c|c|c|c|c|}
\hline $\mathrm{u}_{\mathrm{e}} / \mathrm{c}$ & & 0,1 & & & 0,3 & & & 0,5 & & & 0,7 & & & 0,9 & \\
\hline Case & $I$ & II & III & $I$ & II & III & $I$ & II & III & $I$ & II & III & $I$ & II & III \\
\hline $\mathrm{E}\left[\mathrm{N}_{\mathrm{e}]}\right.$ & 0,868 & 0,892 & 0,856 & 2,350 & 2,358 & 2,345 & 1,980 & 1,938 & 1,990 & 0,877 & 0,884 & 0,852 & 0,243 & 0,250 & 0,241 \\
\hline $\mathrm{E}\left[\mathrm{N}_{\mathrm{s}]}\right.$ & 30,906 & 31,176 & 30,845 & 25,349 & 25,377 & 25,388 & 18,870 & 18,846 & 18,936 & 11,558 & 11,586 & 11,398 & 3,809 & 3,875 & 3,815 \\
\hline$S_{e}$ & 1,925 & 1,999 & 1,898 & 1,660 & 1,671 & \begin{tabular}{l|l|}
1,651 \\
\end{tabular} & 0,806 & 0,789 & 0,813 & 0,254 & 0,256 & 0,246 & 0,055 & 0,056 & 0,055 \\
\hline
\end{tabular}

Table 2: Impact of traffic parameter distribution on $\left(\mathbf{E}\left[N_{e}\right], \mathbf{E}\left[N_{s}\right], S_{e}\right)$ for $\mathbf{P}=\mathbf{2 0 W}$ with various elastic load compositions (I: exponentially distributed $\left(s_{e}, d_{s}\right)$, II: exponentially distributed $d_{s}$ and hyper-exponentially distributed $s_{e}\left(a_{e}=\mathbf{1 0 0}\right)$ and III: exponentially distributed $s_{e}$ and Erlang-2 distributed $\left.d_{s}\right)$.
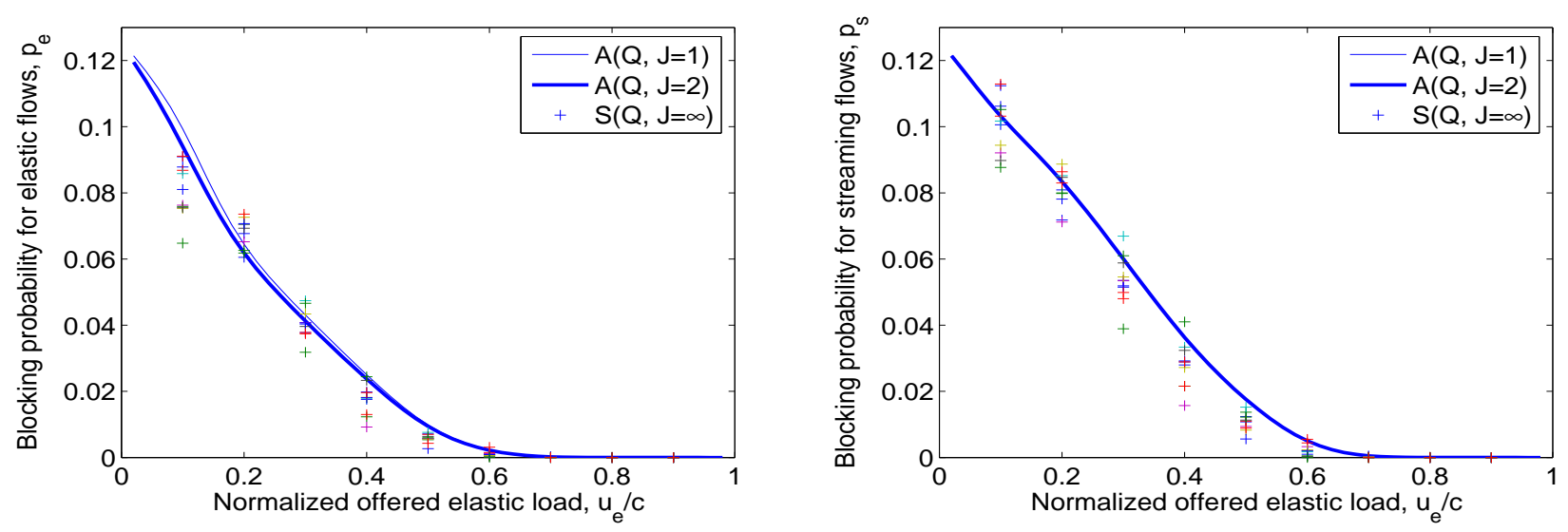

Figure 1: Blocking probability for elastic (left) and streaming requests (right) vs normalized offered elastic load obtained with approximation and simulation for Case I $(\mathrm{P}=\mathbf{2 0 \mathrm { W }})$.

In order to investigate the performance gain with exploiting user location when path-loss is significant, we repeat the simulations for the case of $\mathrm{P}=0.2 \mathrm{~W}$, and plot $\left(\mathrm{E}\left[N_{e}\right], \mathrm{E}\left[N_{s}\right]\right)$ and $\left(p_{e}, S_{e}\right)$ as a function of the traffic mix, $\frac{u_{e}}{c}, 0 \leq u_{e} \leq$ $c$, for $\mathbf{A}(\mathbf{Q}, \mathbf{J})$ in Fig. 2 and 3 respectively. In this case, we note that as cell partitioning becomes finer (increasing $J$ ), the performance obtained with $\mathbf{A}(\mathbf{Q}, \mathbf{J})$ approaches the simulation performance. We expect the accuracy of $\mathbf{A}(\mathbf{Q}, \mathbf{J})$ to be further improved as $J$ is further increased.

\subsection{Performance sensitivity in different traffic regimes}

In the last two sections, we obtained the cell performance through simulations for a quasi-stationary traffic regime, where the dynamics of streaming flows take place on a much slower time scale than those of elastic flows. In particular, we showed in Section 4.3 that $\mathbf{A}(\mathbf{Q}, \mathbf{J}=\mathbf{1})$ accurately approximates the cell performance with $\mathrm{P}=20 \mathrm{~W}$, and when the base station power is reduced to $0.2 \mathrm{~W}$, the accuracy of $\mathbf{A}(\mathbf{Q}, \mathbf{J})$ improves as $J$ is increased.

Here, we define two other traffic regimes: fluid (neutral) traffic regimes, where the dynamics of streaming flows take place on a much faster (similar) time scales than those of elastic flows. Our objective is to investigate if $\mathbf{A}(\mathbf{Q}, \mathbf{J})$ can be applied to these traffic regimes.

We generate 5 sets of simulation results for each scenario, and compute the sample mean for $\left(\mathrm{E}\left[N_{e}\right], S_{e}\right)$. For Case I $(\mathrm{P}=20 \mathrm{~W})$, we plot these metrics as a function of the traffic mix, $\frac{u_{e}}{c}, 0 \leq u_{e} \leq c$, alongside $\mathbf{A}(\mathbf{Q}, \mathbf{J})$ in Fig. 4. We observe that under heavy load condition $\left(\frac{u_{e}}{c} \geq 0.5\right)$, as the load increases, the performance metrics become invariant with respect to the traffic regime. In addition, as expected, the accuracy of $\mathbf{A}(\mathbf{Q}, \mathbf{J})$ is degraded as we move from the quasi-stationary to the neutral regime, and further with the transition into the fluid regime. In this case, $\mathbf{A}(\mathbf{F})$ is necessary to approximate the performance in the latter regime.

We repeat the simulations for Case II $(\mathrm{P}=0.2 \mathrm{~W})$ under moderate loading condition $(\alpha=0.6)$, and the sample means of $\left(\mathrm{E}\left[N_{e}\right], S_{e}\right)$ as a function of the traffic mix in Fig. 5. Accordingly, under reduced power constraints, the performance metrics are almost invariant in the various traffic regimes, and hence, if $\mathbf{A}(\mathbf{Q}, \mathbf{J})$ is sufficiently accurate for the quasistationary regime, it will also be a good approximation for the other traffic regimes.

\section{CONCLUSIONS}

We propose a differentiated admission control strategy for third generation wireless systems that protects users with stringent capacity requirements ("streaming traffic") while offering sufficient capacity over longer time intervals to delay-tolerant users ("elastic traffic").

Since the exact analysis to evaluate the performance of such an integrated services system is non-tractable in general, we apply time-scale decomposition to develop approximations for the cell performance for a single cell scenario.

For the limiting traffic regime (where traffic parameters are such that the dynamics of one traffic type take place at a much finer time scale than the other), the performance in our model is insensitive to traffic parameter distributions, which is in agreement with simulation results. In 

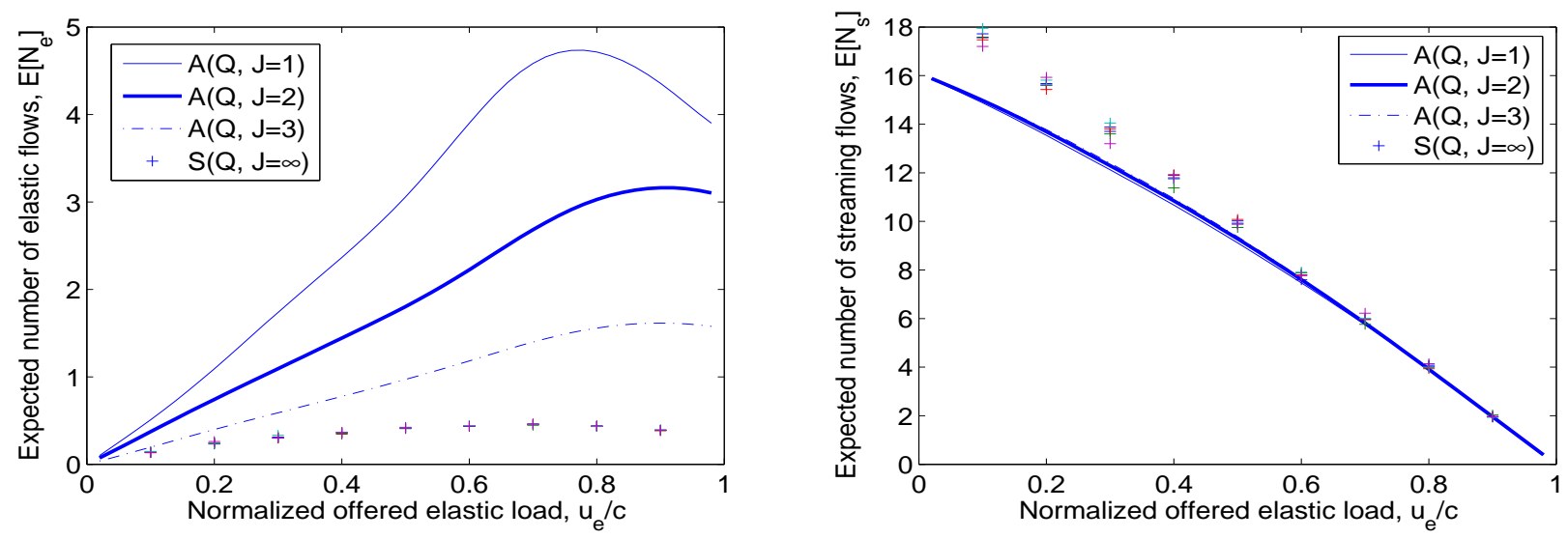

Figure 2: Number of active elastic (left) and streaming (right) requests vs normalized offered elastic load obtained with approximation and simulation for Case $\mathrm{I}(\mathrm{P}=0.2 \mathrm{~W})$.
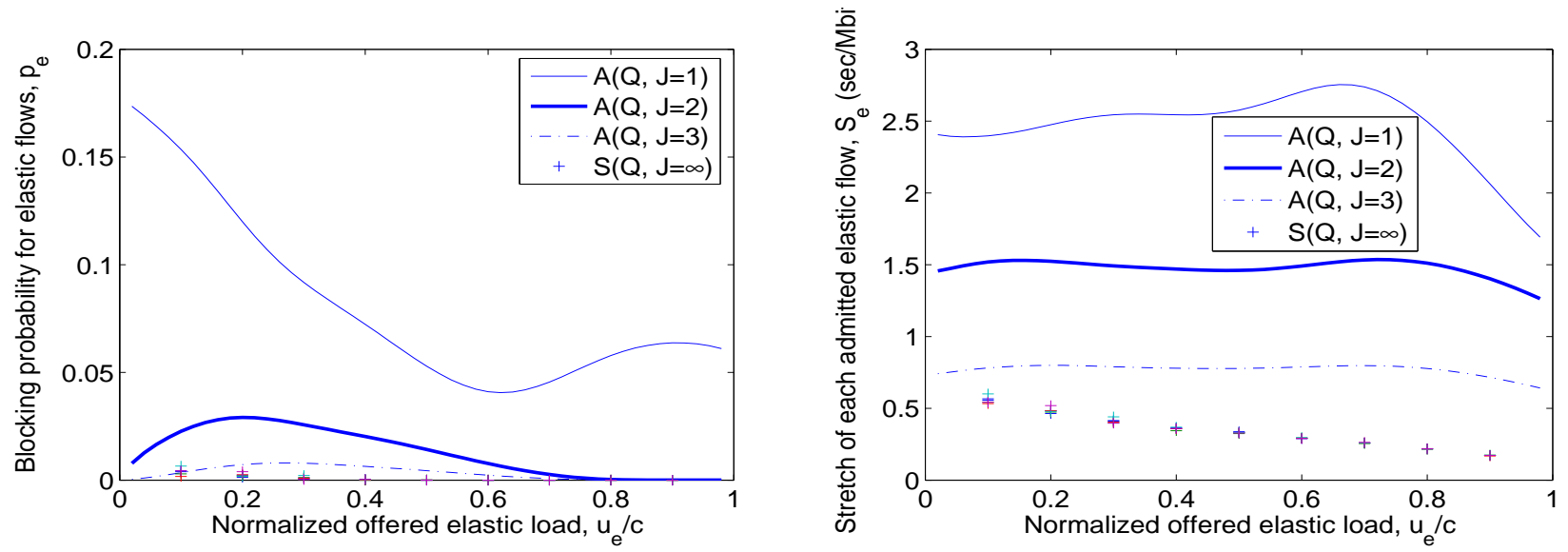

Figure 3: Blocking probability (left) and stretch (right) of elastic requests vs normalized offered elastic load obtained with approximation and simulation for Case I $(P=0.2 \mathrm{~W})$.
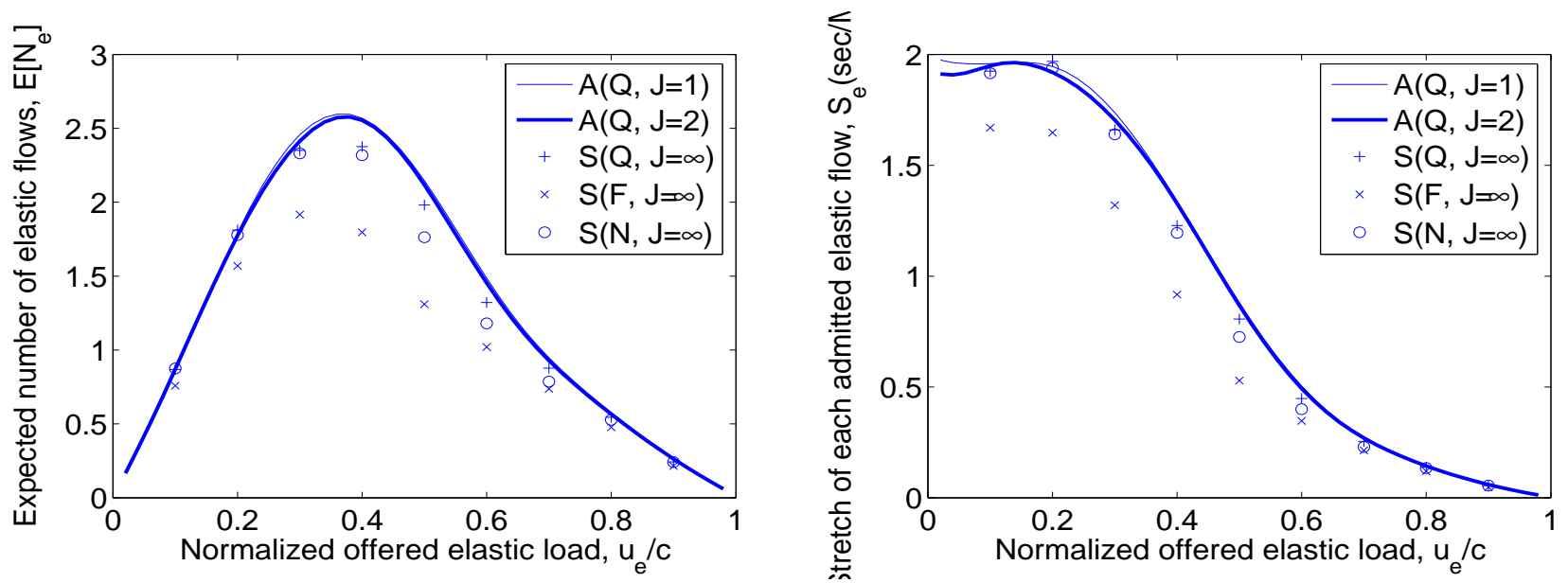

Figure 4: Number of active elastic requests (left) and stretch (right) of each admitted elastic request vs normalized offered elastic load obtained with approximation and simulation in different traffic regimes for Case I $(\mathrm{P}=\mathbf{2 0 W})$. 

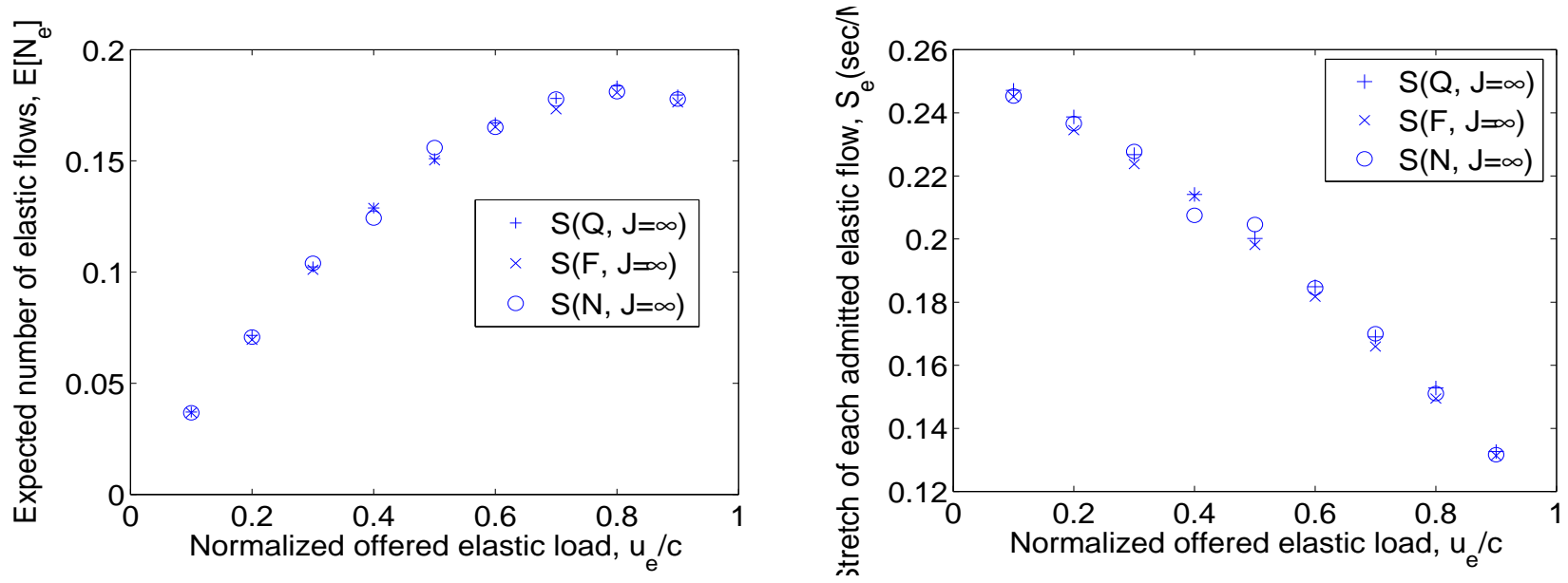

Figure 5: Number of active elastic requests (left) and stretch (right) of each admitted elastic request vs normalized offered elastic load obtained with approximation and simulation in different traffic regimes for Case II $(\mathrm{P}=0.2 \mathrm{~W}, l=0.6)$.

addition, we demonstrate that incorporating asymmetries in channel conditions allows the model to better approximate the cell performance as the cell partitioning becomes finer. The model can be extended so as to incorporate gains from opportunistic scheduling without affecting the model complexity [6].

\section{ACKNOWLEDGMENTS}

The support of Vodafone is gratefully acknowledged. This research is partially supported by the Dutch Bsik/BRICKS project and is performed within the framework of the European Network of Excellence Euro-NGI.

\section{REFERENCES}

[1] N. Benameur, S. B. Fredj, F. Delcoigne,

S. Oueslati-Boulahia, and J. W. Roberts. Integrated admission control for streaming and elastic traffic. LNCS, 2156:69-81, September 2001.

[2] T. Bonald and A. Proutière. Insensitive bandwidth sharing in data networks. Queueing Systems, 44:69-100, 2003.

[3] T. Bonald and A. Proutière. Wireless downlink data channels: User performance and cell dimensioning. Proc. ACM MOBICOM, pages 339-352, September 2003.

[4] T. Bonald and A. Proutière. On performance bounds for the integration of elastic and adaptive streaming flows. Proc. ACM SIGMETRICS / Performance, pages 235-245, June 2004.

[5] S. Borst. User-level performance of channel-aware scheduling algorithms in wireless data networks. IEEE/ACM Transanctions on Networking, pages 636-647, June 2005.

[6] O. J. Boxma, A. F. Gabor, R. Núñez-Queija, and H. P. Tan. Admission control for differentiated services in $3 \mathrm{~g}$ cellular networks. Technical report, 2006. [Online]. Available: http://euridice.tue.nl/ hptan/publications/umts_single_cell.pdf.

[7] O. J. Boxma, A. F. Gabor, R. Núñez-Queija, and H. P. Tan. Performance analysis of admission control for integrated services with minimum rate guarantees. NGI 2006, April 2006.

[8] J. W. Cohen. The multiple phase service network with generalized processor sharing. Acta Informatica, 12:245-284, 1979.

[9] F. Delcoigne, A. Proutière, and G. Regnie. Modeling integration of streaming and data traffic. Performance Evaluation, 55(3-4):185-209, February 2004.

[10] H. Holma and A. Toskala. WCDMA for UMTS, Radio access for third generation mobile communications. John-Wiley and Sons, 2001.

[11] P. Key and L. Massoulié. Fluid Limits and Diffusion Approximations for Integrated Traffic Models. Technical Report MSR-TR-2005-83, Microsoft Research, June 2005.

[12] P. Key, L. Massoulié, A. Bain, and F. Kelly. Fair internet traffic integration: network flow models and analysis. Annales des Telecommunications, 59:1338-1352, 2004.

[13] R. Núñez-Queija. Processor-Sharing Models for Integrated-Services Networks. PhD thesis, Eindhoven University of Technology, 2000.

[14] R. Núñez-Queija, J. L. van den Berg, and M. R. H. Mandjes. Performance evaluation of strategies for integration of elastic and stream traffic. Proc. ITC 16, pages 1039-1050, 1999.

[15] H. C. Tijms. Stochastic Models - An Algorithmic Approach. John-Wiley and Sons, 1994.

[16] Y. Wang and T. Ottosson. Cell search in W-CDMA. IEEE JSAC, 18(8):1470-1482, August 2000. 